\title{
Psychosexual Assessment of Transgender Individuals during the Sex Reassignment Process: Sexual Desire, Activity, and Satisfaction
}

\author{
Carmine Carriero, Miriam Dellino, Francesco Davide Campanelli, Martina Licchelli, \\ Giuseppe Loverro \\ Department of Biomedical Sciences and Human Oncology, University of Bari "Aldo Moro", Bari, Italy \\ Email: carmine.carriero@uniba.it
}

Received 5 July 2016; accepted 21 August 2016; published 24 August 2016

Copyright (C) 2016 by authors and Scientific Research Publishing Inc.

This work is licensed under the Creative Commons Attribution International License (CC BY). http://creativecommons.org/licenses/by/4.0/

(c) (i) Open Access

\section{Abstract}

A group of 39 Transgender subjects, 17 Female to Male (FtM) and 22 Male to Female (MtF), have been evaluated about their sexual desire, activity, and satisfaction during the period before and after Sex Reassignment by Surgery (SRS). Visual Analogue Scale from 0 to 10 has been used to assess sexual desire and satisfaction, number of sexual partners and number of sexual intercourses/ month have been evaluated to assess sexual activity. Moreover, after the surgery, the Sexual Desire Inventory-2 (SDI-2) was administered to patients and related to testosterone (total or free) levels. In FtM, after hysterectomy and bilateral ovariectomy, mean value of sexual desire (VAS 0 10) was 7.5 before beginning testosterone therapy, this value increased after six months of therapy and after one year. Just before SRS the value decreased, but increased to 8.42 after SRS. In the same patients the values (VAS 0 - 10) on sexual satisfaction were respectively $6.5,6.9,7.1,6.5$ and 7. Mean number of partners was 4, with 7.07 mean frequency of sexual intercourse/month, before starting therapy (after hysterectomy). During hormonal therapy (testosterone), No. of partners was 2.46 and 8.96 intercourses/month, while after SRS the partners were 1.25 and intercouses/month 7.91. MtF patients reported a sexual desire (VAS 0 - 10) before therapy of 7, this remained almost constant after 6 months, decreases after 12 months and just before surgery, and finally increased to $\mathbf{7 . 7 6}$ after SRS. For sexual satisfaction mean values of VAS were respectively: 6.1, 6.2, 6.3, 6, and 6.8. Regarding sexual activity, MtF report 3.81 mean No. of partners and 9.27 intercouses/month before the beginning of hormonal therapy, 1.36 partners and 4.36 intercourses after therapy, and 1.3 partners and 4.54 intercourses after SRS. 


\section{Keywords}

\section{Transgender, Sexuality, Sexual Desire, Sexual Satisfaction, Sex Reassignment Surgery}

Finally, the comparative evaluation of SDI-2 results and hormonal levels after the Sex Reassignment by Surgery shows a significant correlation $(\mathrm{p}<0.0012)$ between total testosterone and SDI-2 value in FtM subjects, while free-testosterone was significantly related to SDI-2 value in MtF $(\mathrm{p}<0.00001)$. The linear regression shows the direct correlation between testosterone and Sexual Desire Inventory results. No significant correlation has been found between other hormones (estrogens, FSH, LH) and SHBG level and sexual desire.

\section{Introduction}

It is essential to consider the concept of sexual identity by operators, physicians or psychologists, who care the health and the psychophysical wellness of gay, lesbian, bisexual, and transgender individuals. Therefore, a Centre for Gender Identity Disorder (GID) has been created in the Department of Gynecology and Obstetrics of the University Hospital of Bari. In this Centre, from 2010, amultidisciplinary approach is followed to care patients with GID, mainly by four specialists, such as endocrinologist, gynaecologist, urologist, and psychiatrist (sexologist), and other consultants, like cardiologist, plastic surgeon, etc.

The issue of sexual activity in these patients (before and after surgery) is considered a not negligible aspect, considering changes in sexual desire, activity, and satisfaction due to gender reassignment by hormonal and/or surgical treatment, with a big impact on wellness and quality of life. This problem about sexual activity and sexual desire is well known by transgender candidates, together with the irreversible loss of reproductive capacity [1]. Failure of surgical procedure or post-operative complications may cause sexual dysfunction or impairment, such as, in Female to Male (FtM), suture dehiscence, lack of symmetry of mammalian areoles post mastectomy, recto-vesical fistula; and, in Male to Female (MtF), stenosis of neovagina, recto-vesical fistula, unsatisfactory esthetical aspect of neovulva or mastoplasty [2]. In MtF subjects social dynamics, that compromise the post surgical general healing and recovery of life, are associated, such as non-acceptance of sex reassignment by the partner, more difficult social integration, persistence of the archaic concept that giving up virility is less acceptable than giving up femininity.

Therefore, MtFpost surgery patients often show a depressive emotional status, that is definitively compromising the sexual activity [3].

The observation of these dynamics encouraged us to re-evaluate sexuality during follow up of both MtF and FtM patients.

The aim of this study has been concentrated on the following points:

- to evaluate sexual desire variations by standard methods in both MtF and FtM patients;

- to verify a possible correlation between sexuality and hormonal status and treatment in transgender subjects;

- to evaluate the different impact on sexuality during sex reassignment in MtF and FtM patients.

\section{Material and Methods}

Forty-eight patients have been invited to participate to this study, in whom the diagnosis of "Gender Dysphoria" was confirmed according to the diagnostic criteria of DSM-5 [4]: it replaces the diagnostic name "gender identity disorder" of DSM-4 with "gender dysphoria" as well as makes other important clarifications in the criteria. It is important to note that gender nonconformity is not in itself a mental disorder. The critical element of gender dysphoria is the presence of clinically significant distress associated with the condition. There must be a marked difference between the individual's expressed/experienced gender and the gender others would assign him or her, and it must continue for at least six months. The DSM-5 diagnosis adds a post-transition specifier for people who are living full-time as the desired gender (with or without legal sanction of the gender change). This ensures treatment access for individuals who continue to undergo hormone therapy, related surgery, or psycho- therapy or counselling to support their gender transition.

In particular, the population of the study is subdivided into two groups

- 17/39 FtM, mean age 31 (range 26 - 37), already treated by surgery, followed by hormonal therapy, and then 
SRS (Sex Reassignment by Surgery);

- 22/39 MtF, mean age 35 (range 28 - 40), after SRS and with hormonal therapy.

During the follow up period, some critical moments have been identified, such as:

- before starting hormonal therapy;

- six months after the beginning of hormonal therapy;

- one year after hormonal therapy;

- one month before surgical reassignment of sex;

- post surgical period.

Follow up every six months have been scheduled for all two groups, including:

- blood tests, comprehensive of hormonal levels;

- DEXA (bone densitometry), mammography, ECG, to evaluate side effects or complications of hormonal therapy;

- evaluation of sexual desire, sexual activity, and sexual satisfaction.

To evaluate sexual desire and sexual satisfaction we used a VAS (Visual Analogue Scale from 0 to 10), during all follow-up events.

For sexual activity evaluation we asked patients about number of partners and frequency of sexual intercourses (n./month).

At the end of follow-up, for a more specific correlation of hormonal and SRS effects with sexual desire, we used for all TG subjects the SDI-2 (Sexual Desire Inventory-2), that is a brief 14-item scale that aims to measure the multidimensional construct of sexual desire in a dyadic context. Four items are scored on an 8-item response scale from "0" (= "not at all") to "7" (= "more than once a day") concerning frequency of desire. The remaining items were answered on a 9-point Likert scale ranging from "0" (= "no desire") to "8" (= "strong desire"). Possible score range from 0 to 112 [5].

Therefore, a correlation between SDI-2 results and levels of total testosterone, free testosterone, estradiol, FSH, LH, SHBG has been verified in both FtM and MtF subjects.

Statistical analysis: due to the small numbers of the sample and non-parametric nature of the variables (sexual desire and satisfaction) the use of VAS from 0 to 10 allowed only a descriptional observation of the subjective feelings of the patients about their sexuality in different phases of the follow up. For the correlation between hormonal levels and sexual desire at the end of follow-up, the results of SDI-2 were related to hormonal values by linear regression and p value.

\section{Results}

In FtM, after hysterectomy and bilateral ovariectomy, mean value of sexual desire (VAS 0 - 10) was 7.5 before beginning testosterone therapy, 8.35 after six months of therapy and 8.57 after one year. Just before SRS the value decreases to 7.92, but increases to 8.42 after SRS. In the same patients the values (VAS 0 - 10) on sexual satisfaction were respectively 6.5, 6.9, 7.1, 6.5, and 7 (see Figure 1). In this group, mean number of partners was 4, with 7.07 mean frequency of sexual intercourse/month, before starting therapy (after hysterectomy). During hormonal therapy (testosterone) n. of partners was 2.46 and 8.96 intercourses/month, while after SRS the partners were 1.25 and intercouses/month 7.91 (Figure 2).

MtF patients report a sexual desire (VAS 0 - 10) before therapy of 7, this remains almost constant (7.07) after 6 months, decreases to 6.76 after 12 months and just before surgery, and finally increases to 7.76 after SRS. For sexual satisfaction mean values of VAS were respectively: 6.1, 6.2, 6.3, 6, and 6.8 (Figure 3). Regarding sexual activity, MtF report 3.81 mean $\mathrm{n}$. of partners and 9.27 intercouses/month before the beginning of hormonal therapy, 1.36 partners and 4.36 intercourses after therapy, and 1.3 partners and 4.54 intercourse after SRS (Figure 4).

Finally, the comparative evaluation of SDI-2 results and hormonal levels after the Sex Reassignment by Surgery shows a significant correlation $(\mathrm{p}<0.0012)$ between total testosterone and SDI-2 value in FtM subjects, while free-testosterone was significantly related to SDI-2 value in $\operatorname{MtF}(\mathrm{p}<0.00001)$. In Figure 5 the linear regression shows the direct correlation between testosterone and Sexual Desire Inventory results. No significant correlation has been found between other hormones (oestrogens, FSH, LH) and SHBG level and sexual desire.

\section{Discussion}

From results of this study it is evident an increase of sexual desire during the therapeutic process for FtM patients, 


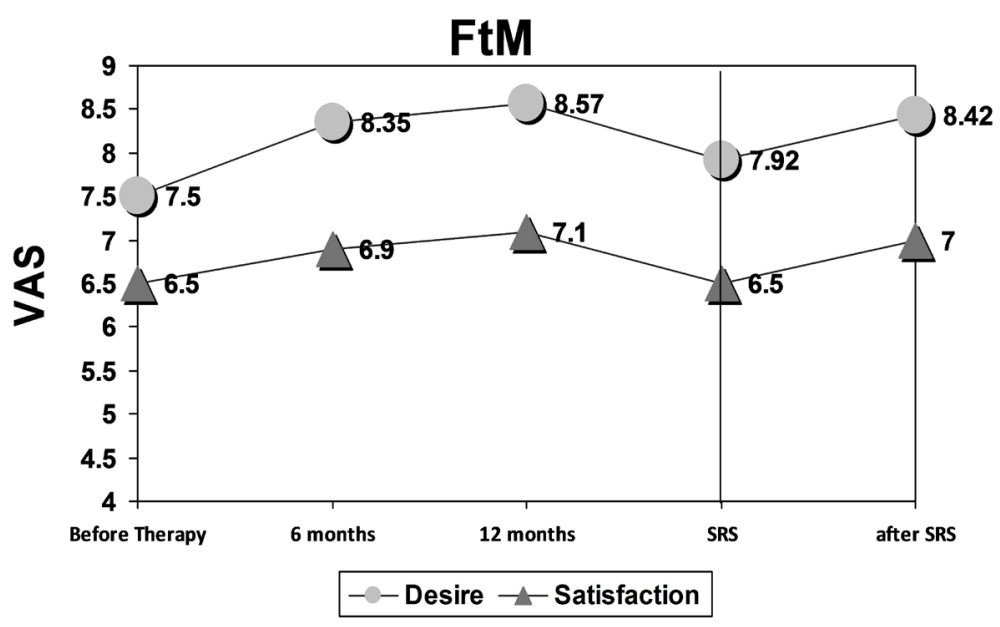

Figure 1. Mean values of VAS (Visual Analogue Scale 0 - 10) about sexual desire and sexual satisfaction in FtM.

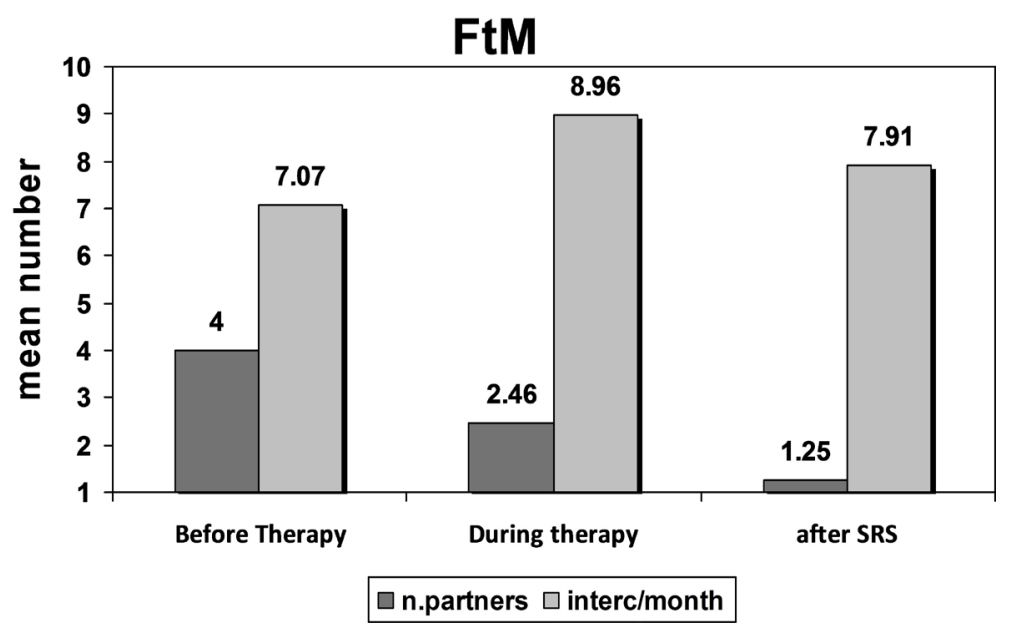

Figure 2. Mean number of sexual partners and intercourses/month in FtM patients.

MtF

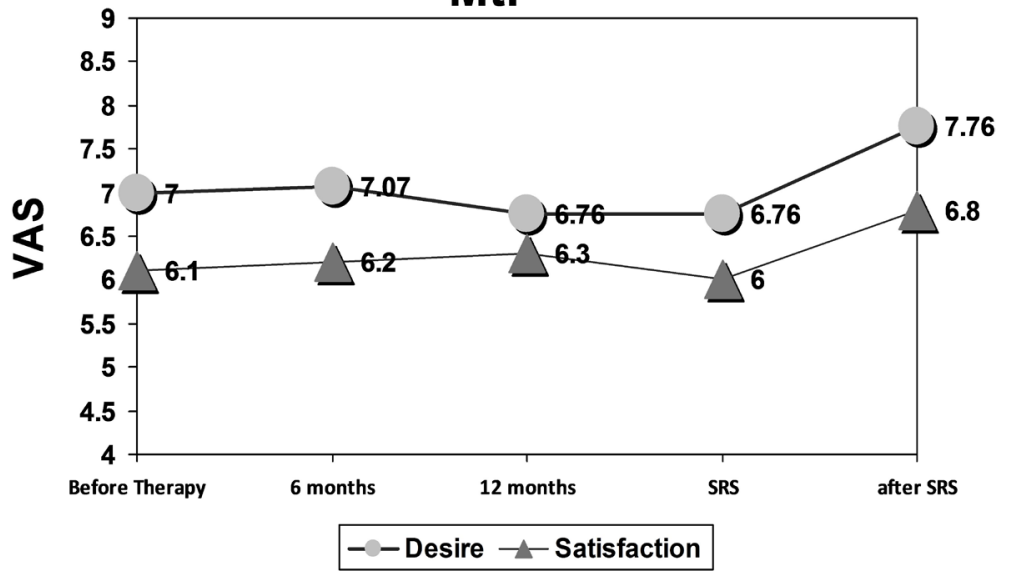

Figure 3. Mean values of VAS (Visual Analogue Scale 0 - 10) about sexual desire and sexual satisfaction in $\mathrm{MtF}$. 


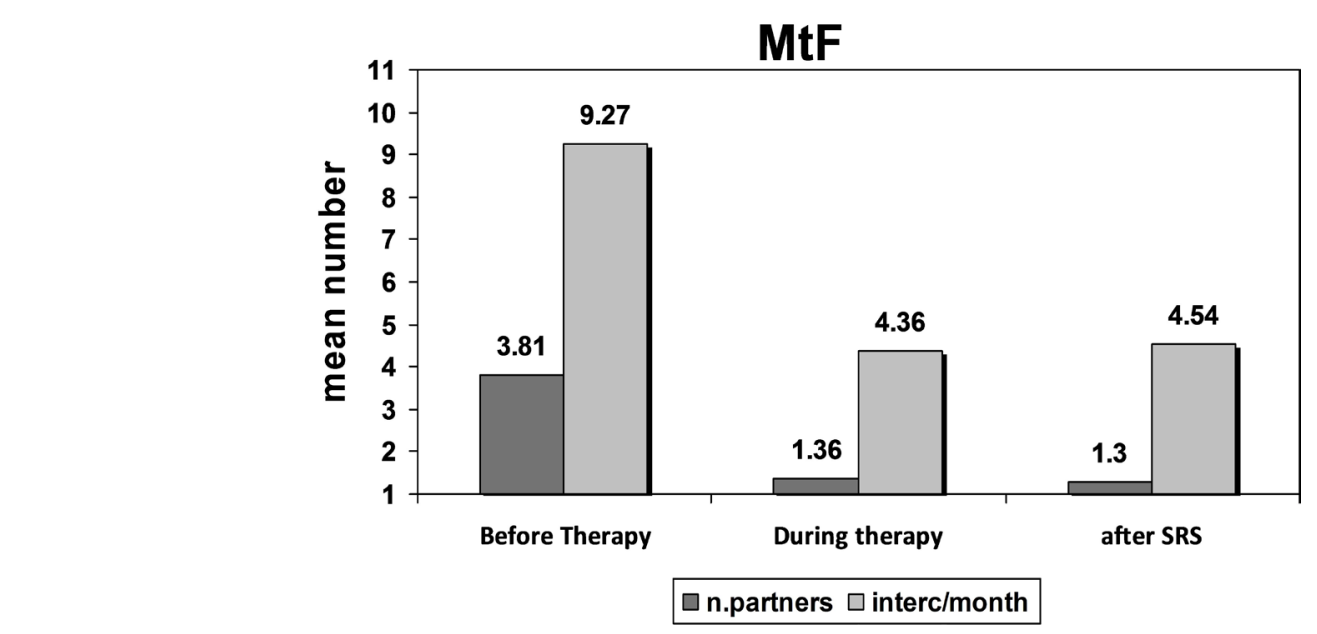

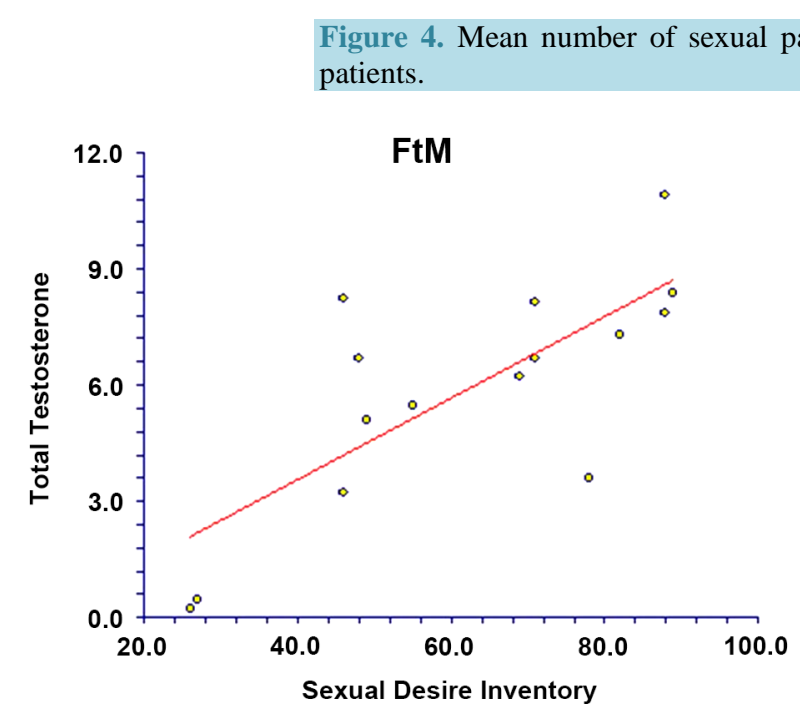

(a)

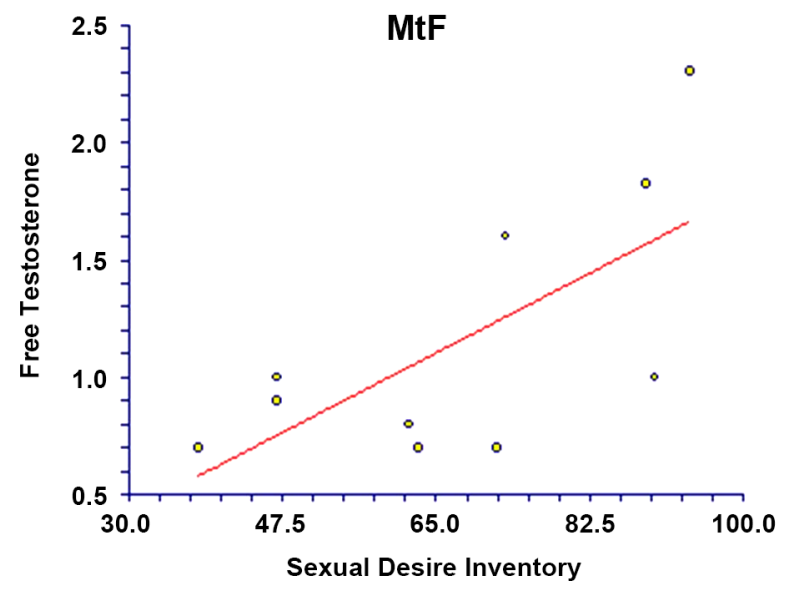

(b)

Figure 5. Linear regression shows direct correlation between sexualdesire and Testosterone (ng/dl).

while MtF experience a decrease of desire, that recuperates after the SRS.

In literature the positive effect of testosterone on sexual desire is well documented [6] [7], our sample confirms the increased sexual desire after testosterone administration and its decreasing when testosterone is interrupted.

The therapeutic path towards SRS is a fundamental turning point on sexuality variation for at least two reasons: firstly, the subject must realize what is going to happen on his/her body, and this is significantly influencing psyche, so that sexual desire and activity are strongly conditioned. Secondly, just before SRS hormonal therapy is interrupted, and this is causing alteration of a balance, which is instable in itself, In fact, in this phase there is a fall of sexual desire, that recoveries to pre-surgery levels, when there is an acceptation of his/her own body, and hormonal support is restored.

Sexual desire is a motivational state and an interest in "sexual objects or activities, or as a wish, need, or drive to seek out sexual objects or to engage in sexual activities" [8]. Synonyms for sexual desire are libido, sexual attraction, and lust. [9] Sexual desire is an aspect of a person's sexuality, which varies significantly from one person to another, and also varies depending on circumstances at a particular time.

Sexual desire is a subjective feeling state that can "be triggered by both internal and external cues, and that may or may not result in overt sexual behaviour" [10]. Sexual desire can be spontaneous or responsive, is dynamic, can either be positive or negative, and can vary in intensity depending on the desired object/person. The sexual desire spectrum is described by Stephen B. Levine as: aversion $\rightarrow$ disinclination $\rightarrow$ indifference $\rightarrow$ in- 
terest $\rightarrow$ need $\rightarrow$ passion. Levine suggests that sexual desire has three components, which link several different theoretical perspectives together: 1) Drive-the biological component, this includes anatomy and neuroendocrine physiology. 2) Motivation-the psychological component, this includes the influences of personal mental states (mood), interpersonal states (e.g. mutual affection, disagreement), and social context (e.g. relationship status). 3) Wish - the cultural component, this considers cultural ideals, values, and rules about sexual expression, which are external to the individual [11].

There are very few recent studies dealing with the issue of sexuality in transgender people. In a study of 2016 [12] interviews were conducted with 16 heterosexual men who reported at least one sexual encounter with a transgender woman in the previous 12 months. Using principles of Grounded Theory, three themes emerged: 1) the erotic desire that transpired from a transgender woman's construction of her femininity, 2) the sexual act that dictated the specific navigation of a transgender woman's penis and 3) the sexual dissonance that resulted from being a heterosexually identified man having sex with a partner who had a penis. An ethnographic study of MSTW (Men who have sex with transgender women) in New York City was conducted between December 2005 and May 2007 [13]. The specific objectives were to: 1) describe the sex marketplaces and the sexual experiences of an ethnographic sample of MSTW in New York City and 2) describe the ways MSTW construct their sexual partnering practices and the meanings attributed to those practices in relation to varying social contexts (in and outside the sex marketplace). In this analysis were identified three major recurrent themes in the ways that MSTW organized their sexual desire for TW transitioning from sex marketplaces to social spaces in their lives: 1) phallus-centric trade sex market focus; 2) relational-companionship marketfocus; and 3) specialized market focus. Another study was conducted online [14], with the aim of exploring the gender identities, sexual orientation identities, and surgery and hormonal statuses of those who identify with a gender identity other than, or in addition to, that associated with their birth sex $(n=292)$. Genderqueer was the most commonly endorsed gender identity, and pansexual and queer were the most commonly endorsed sexual orientation identities.

Factors that may influence sexual desire are social and relationship influences, religious views, mental disorders, physical health, medications, and hormones.

In the very special situation of transgender individuals, influence of psychosocial factors cannot be denied: on one side the social prejudice that tends to stigmatize these subjects, on the other side the progressive acceptance of their own new gender identity conducts them to more freely live their sexuality.

In our study sexual activity shows halving of partners, but doubling of sexual intercourses per month. In MtF sexual desire slowly declines until surgery, then increases after SRS. Considering sexual activity, reduction of number of partners and sexual intercourses are coincident, and reduction of sexual activity remains after SRS, differently from FtM patients. Fall of desire is also justified by antiandrogens use and gonadectomy, the parallel reduction of intercourses and sexual satisfaction is linked to more difficult social integration of MtF, compared with FtM, being loss of virility much more stigmatized than loss of femininity. Anyway, both MtF and FtM tend to reduce number of partners and to achieve more sentimental stability and monogamic relationship, with a partner who accepted gender reassignment.

\section{Conclusions}

Sexual desire is a sum of many aspects and its objective measurement is difficult to obtain. Adoption of Visual Analogue Scale and Sexual Desire Inventory may help in this particular population affected by "gender dysphoria”, who demonstrates a good compliance of sexual investigation and analysis. Transgender individuals showed the aspiration to achieve not only the "right" gender but also a stable and monogamic relationship with the "right" partner.

Having care of this population needs a correct balance between psychosocial and relational aspects on one side and hormonal and biological evaluations on the other side. A multidisciplinary approach is then mandatory for these patients.

\section{References}

[1] Wierckx, K., Elaut, E., Van Hoorde, B., Heylens, G., De Cuypere, G., Monstrey, S., Weyers, S., Hoebeke, P. and T'Sjoen, G. (2014) Sexual Desire in Trans Persons: Associations with Sex Reassignment Treatment. The Journal of Sexual Medicine, 11, 107-118. http://dx.doi.org/10.1111/jsm.12365 
[2] Kronawitter, D., Gooren, L.J., Zollver, H., Oppelt, P.G., Beckmann, M.W., Dittrich, R. and Mueller, A. (2009) Effects of Transdermal Testosterone or Oral Dydrogesterone on Hypoactive Sexual Desire Disorder in Transsexual Women: Results of a Pilot Study. European Journal of Endocrinology, 161, 363-368. http://dx.doi.org/10.1530/EJE-09-0265

[3] Elaut, E., Bogaert, V., De Cuypere, G., Weyers, S., Gijs, L., Kaufman, J. and T’sjoen, G. (2010) Contribution of Androgen Receptor Sensitivity to the Relation between Testosterone and Sexual Desire: An Exploration in Male-to-Female Transsexuals. Journal of Endocrinological Investigation, 33, 37-41. http://dx.doi.org/10.1007/BF03346547

[4] Regier, D.A., Kuhl, E.A. and Kupfer, D.J. (2013) The DSM-5: Classification and Criteria Changes. World Psychiatry, 12, 92-98. http://dx.doi.org/10.1002/wps.20050

[5] Spector, I.P., Carey, M.P. and Steinberg, L. (1996) The Sexual Desire Inventory: Development, Factor, Structure and Evidence of Reliability. Journal of Sex \& Marital Therapy, 22, 175-190. http://dx.doi.org/10.1080/00926239608414655

[6] Costantino, A., Cerpolini, S., Alvisi, S., Morselli, P.G., Venturoli, S. and Meriggiola, M.C. (2013) A Prospective Study on Sexual Function and Mood in Female-to-Male Transsexuals during Testosterone Administration and after Sex Reassignment Surgery. Journal of Sex \& Marital Therapy, 39, 321-335. http://dx.doi.org/10.1080/0092623X.2012.736920

[7] Wierckx, K., Elaut, E., Van Caenegem, E., Van De Peer, F., Dedecker, D., Van Houdenhove, E. and T’Sjoen, G. (2011) Sexual Desire in Female-to-Male Transsexual Persons: Exploration of the Role of Testosterone Administration. European Journal of Endocrinology, 165, 331-337. http://dx.doi.org/10.1530/EJE-11-0250

[8] Regan, P.C. and Atkins, L. (2006) Sex Differences and Similarities in Frequency and Intensity of Sexual Desire. Social Behavior \& Personality: An International Journal, 34, 95-101. http://dx.doi.org/10.2224/sbp.2006.34.1.95

[9] Spector, I.P., Carey, M.P. and Steinberg, L. (1996) The Sexual Desire Inventory: Development, Factor Structure, and Evidence of Reliability. Journal of Sex \& Marital Therapy, 22, 175. http://dx.doi.org/10.1080/00926239608414655

[10] Basson, R. (2000) The Female Sexual Response: A Different Model. Journal of Sex \& Marital Therapy, 26, 51-65. http://dx.doi.org/10.1080/009262300278641

[11] Levine, S.B. (2003) The Nature of Sexual Desire: A Clinician’s Perspective. Archives of Sexual Behavior, 32, $279-285$. http://dx.doi.org/10.1023/A:1023421819465

[12] Reback, C.J., Kaplan, R.L., Bettcher, T.M. and Larkins, S. (2016) The Role of the Illusion in the Construction of Erotic Desire: Narratives from Heterosexual Men Who Have Occasional Sex with Transgender Women. Culture, Health \& Sexuality, 18, 951-963. http://dx.doi.org/10.1080/13691058.2016.1150515

[13] Mauk, D., Perry, A. and Muñoz-Laboy, M. (2013) Exploring the Desires and Sexual Culture of Men Who Have Sex with Male-to-Female Transgender Women. Archives of Sexual Behavior, 42, 793-803. http://dx.doi.org/10.1007/s10508-013-0079-z

[14] Kuper, L.E., Nussbaum, R. and Mustanski, B. (2012) Exploring the Diversity of Gender and Sexual Orientation Identities in an Online Sample of Transgender Individuals. Journal of Sex Research, 49, 244-254. http://dx.doi.org/10.1080/00224499.2011.596954

\section{Submit or recommend next manuscript to SCIRP and we will provide best service for you:}

Accepting pre-submission inquiries through Email, Facebook, LinkedIn, Twitter, etc.

A wide selection of journals (inclusive of 9 subjects, more than 200 journals)

Providing 24-hour high-quality service

User-friendly online submission system

Fair and swift peer-review system

Efficient typesetting and proofreading procedure

Display of the result of downloads and visits, as well as the number of cited articles

Maximum dissemination of your research work

Submit your manuscript at: http://papersubmission.scirp.org/ 